\title{
Analysis of Clinical, Laboratory and Bone Marrow Features in Dominant TPO, MPL and JAK2 Germline Mutated Hereditary Essential Thrombocythemia (HET) Versus Acquired MPL ${ }^{515}$, CALR and JAK2 ${ }^{\mathrm{V} 617 \mathrm{~F}}$ Mutated ET in Myeloproliferative Neoplasms
}

\section{Jan Jacques Michiels ${ }^{1 *}$, Hendrik De Raeve², Fibo Ten Kate ${ }^{3}$, King Lam ${ }^{3}$, Achille Pich ${ }^{4}$, Vincent Potters ${ }^{5}$ and Francisca Valster $^{5}$}

${ }^{1}$ Bloodcoagulation Specialist, Hematologist and Scientific Investigator. Goodheart Institute and Foundation in Nature Medicine and Health, Freedom of Science and Education on behave of the International Collaborations and Academic Research in Myeloproliferative Neoplasms (ICAR.MPN)

${ }^{2}$ Department of Pathology OLV Hospital Aalst, University Hospital Brussels, Belgium

${ }^{3}$ Department of Pathology, Erasmus University Medical Center, Rotterdam, Netherlands

${ }^{4}$ Department of Molecular Biotechnology and Health Sciences, Section of Pathology, University of Turin

${ }^{5}$ Departments of Pathology and Hematology, BRAVIS Hospital, Bergen op Zoom, Netherlands

*Corresponding Author: Jan Jacques Michiels, Bloodcoagulation Specialist, Hematologist and Scientific Investigator. Goodheart Institute and Foundation in Nature Medicine and Health, Freedom of Science and Education on behave of the International Collaborations and Academic Research in Myeloproliferative Neoplasms (ICAR.MPN).

Received: October 03, 2019; Published: October 22, 2019

DOI: 10.31080/ASMS.2019.03.0444

\begin{abstract}
Heterozygous germline gain of function mutation in the TPO gene induces increased levels of plasma TPO as the cause of dominant hereditary essential thrombocythemia (HET) in two families. Gain of function mutations of the Thrombopoietine (TPO) receptor/myeloproliferative leukemia (MPL) receptor in congenital dominant MPL ${ }^{\text {S205N }}$ mutated HET and acquired MPL ${ }^{515}$ mutated ET are driver causes of normocellular ET without features of PV. The clinical spectrum of acquired JAK2 ${ }^{\mathrm{V} 617 \mathrm{~F}}$ mutated ET patients is featured by by loose clustered pleiomorphic megakaryocytes in normocellular bone marrow with local increase of erythropiesis, spontaneous endogenous erythroid colony formation (EEC), increased leukocyte alkaline phohatase (LAP) score and low serum EPO levels consistent with prodromal PV. Each of the three variants of dominant heterozygous germline JAK2 ${ }^{\mathrm{V} 6171}$, JAK2 ${ }^{\mathrm{R} 564 \mathrm{Q}}$ and JAK2 ${ }^{\mathrm{H} 608 \mathrm{~N}}$ mutated HET does induce cytokine hyperresponsiveness of the hematopoietic progenitor cells to TPO as the cause HET phenotype in blood and bone marrow with normal EEC, plasma TPO and serum EPO levels indicating the absence of PV features. EEC, LAP score and serum EPO levels are normal in MPL and CALR mutated ET and in TPO and JAK2 germline mutated HET patients. Dominant TPO, MPL and JAK2 germline mutated HET, acquired JAK2 ${ }^{\mathrm{V} 617 \mathrm{~F}}$ ET and MPL ${ }^{515}$ mutated ET patients present with aspirin responsive Erythromelalgic Thrombotic Thrombocythemia (ETT) as a novel platelet microvascular thrombophilia in thrombocythemia at platelet count between 400 to $1000 \times 10^{9} / \mathrm{L}$ or above. ETT was not recorded in CALR mutated ET at platelet counts between 400 and $1000 \times 10^{9} / \mathrm{L}$.
\end{abstract}

Keywords: Thrombocythemia; Myeloproliferative; Neoplasms; Germline

\section{Introduction}

Evidence for a decisive role of upregulated TPO in ET became available in the 1990s from observations in mice overexpressing a TPO transgene where increased TPO production resulted in a fatal myeloproliferative disorder [1]. High dose exposure to TPO in lethally irradiated mice grafted with bone marrow cells infected with a retrovirus carrying the murin TPO cDNA (TPO ${ }^{\text {high }}$ mice) developed a lethal myeloproliferative disorder of TPO induced megakaryocytic granulocytic myeloproliferation with reduced erythropoiesis in the spleen and bone marrow [2]. Normal wild type mice respond to TPO treatment by increasing the number of platelets in the circulation and megakaryocytes in the spleen at day 7 to 10 and returned to pretreatment values at day 14 [3]. TPO treatment increased platelet counts 2.3 fold and increased number of megakaryocytes and CFU-Mks. TPO treatment had profound effects on the change of normal into large sized immature megakaryocyte morphology in wild type mice. TPO treatment of wild type mice induced decreased GATA-1 content in megakaryocytes followed by myelofibrosis associated with high levels of transforming growth factor beta-1 (TGG-Beta ${ }^{-1}$ ) expression in bone marrow and spleen 
[3]. Continuous forced expression of TPO, (TPO high mice) in mice induces megakaryocyte proliferation and differentiation and subsequently develop large spleen bone marrow fibrosis $[1,2]$. TPO high mice engineered to overexpress TPO in their liver and those that received transplants of marrow cells infected with a TPO containing retrovirus develop thrombocythemia with massive bone marow hyperplasia of megakaryocytes and granulocytes and hypoplasia of erythropoiesis followed by myelofibrosis and extramedullary hematopoiesis within 2 to 3 months and die from myelofibrosis thereafter [1]. Megakaryocytes from TPO ${ }^{\text {high }}$ rats and mice express high levels of TGF-beta-1 (TGFB1) in marrow extracellular fluids and plasma [3]. In wild mice TGFB1 mRNA expression in bone marrow and spleen was barely detectable before TPO treatment, and significantly increased in both organs after TPO treatment and returned to basal levels at day 14 [3]. Another growth factor produced by megakaryocytes, platelet derived growth factor (PDGF) was found to be upregulated in a fashion similar to TGFB1. High levels of TGFB1 mRNA in bone marrow and spleen cells in TPO high mice were associated with high levels of TGF-beta1 protein in extracellular fluids from these organs. These experimental data on TPO in wild type mice in the1990s predicted mutations in the TPO gene and TPO-Receptor (MPL) as candidate causes for essential thrombocythemia.

\section{TPO mutated hereditary essential thrombocythemia: HET}

An activating mutation in the TPO gene that causes hereditary essential thrombocythemia (HET) has been discovered by Skoda by the demonstration that the co-segregation of $\mathrm{G}$ to $\mathrm{C}$ transversion in the splice donor site of intron 3 in the TPO gene as the cause of dominant essential thrombocythemia (ET) in the Dutch HET family (Figure 1) [4,5]. The propositus case II3 of the Dutch HET family presented in 1986 with typical erythromelalgia complicated by acrocyanosis of a few toes followed by gangrene and amputation of toe (Figure 1). Recurrent erythromelalgia and acrocyanosis in 1986 typically responded to low dose aspirin but not to coumadin in the affected members of the Dutch HET family similar as has been first demonstrated by Michiels., et al. in 1985 for erythromelalgia caused by platelet mediated arteriolar inflammation and thrombosis in acquired ET [4,5]. Bone marrow histology of the propositus of the Dutch HET family (case II 3, figures 1 at age of 52 years) in 1986 showed increase of clustered large mature megakaryocytes compatible with ET and similar to increase of large megakaryocytes in acquired ET complicated by erythromelalgia in thrombocythemia of ET and polycythemia vera (PV) patients [4]. Follow-up bone marrow histopathology from the propositus at age 57 in 1991 (Figure 2) was very characteristic and diagnostic for ET. All features according to the 1980 Rotterdam diagnostic criteria (RCP) of ET proposed by the Thrombocythemia Vera Study Group (TVSG) [6,7], were present affected members of the Dutch HET family: 1: Increase of platelet count in excess of $400 \times 10^{9} / 1$ in the absence of any cause or sign of reactive thrombocytosis. 2: Typi- cally clustering and increase of enlarged megakaryocytes showing mature cytoplasm and hyperploid nuclei in a normocellular bone marrow. 3: No preceding or allied other subtype of myeloproliferative disorder (MPD) or myelodysplastic syndrome (MDS). 4: Normal cellularity of the bone marrow with only slight increase of fine reticulin fibers.

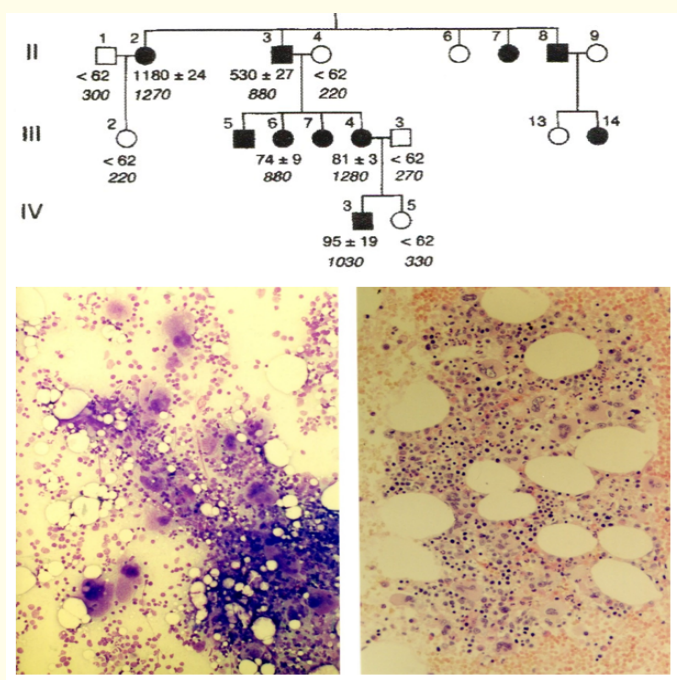

Figure 1: Dutch family with hereditary essential thrombocythemia (HET) caused by a gain of function mutation in the TPO gene with increased values for plasma TPO (normal value $<62 \mathrm{pg} / \mathrm{mL}$ ) and increased platelet counts (normal values $<350 \times 10^{9} / \mathrm{L}$ ) $[5,9]$. Filled in symbols affected individuals, open

symbols normal individuals. Lower part. Bone marrow morphology in smears and histology from biopsy (1986) in the propositus C3 of the Dutch HET family showing a normocellular bone marrow with increase and clustering of large mature megakaryocytes with hyperlobulated nuclei, and no significant increase of reticulin fibers (RF 0/1). Dr Ten Kate, Pathology

Laboratory, Erasmus University Medical Center.
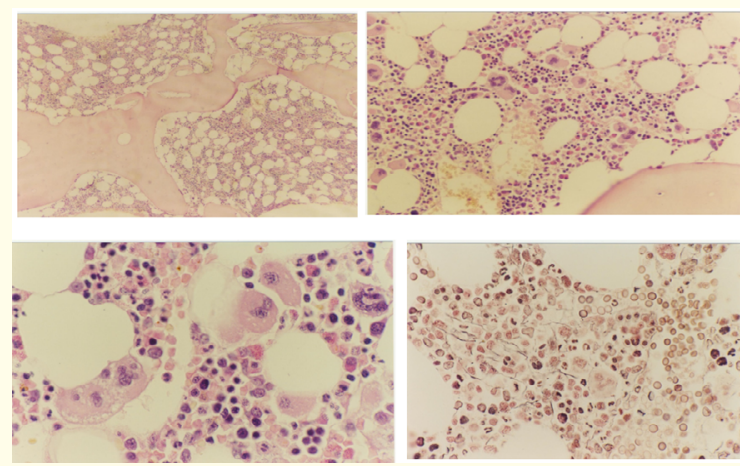

Figure 2: Bone marrow morphology (1991) showing increase and clustering of large megakaryocytes in bone marrow smear (left) and biopsy (right) in the propositus C3 of the Dutch HET family caused by a gain of function mutation in the TPO gene. Dr Ten Kate, Pathology Laboratory, Erasmus Universty Medical Center. 
A second large HET family in Poland studied by Skoda was caused by an identical mutation $\mathrm{C} \rightarrow \mathrm{G}$ transversion in the splice donor of intron 3 of the THPO gene similar as in the dutch HET family [8]. The clinical presentation in 11 affected members of the Polish HET family was associated with a increased frequency of aspirin sensitive microvascular circulation disturbances due to increased platelet counts between 408 and $1340 \times 10^{9} / \mathrm{L}$ with normal leukocyte and erythrocyte counts, no or minor splenomegaly (Table 1). Bone marrow histology revealed an increase of clustered megakaryopoiesis in a normocellular bone marrow with normal myeloid/ erythroid ratio and absence of EEC (Figure 3). The bone marrow histology of affected members of the Polish HET family was consistent with pefibrotic stage of ET featured by: 1. increase and loose to dense clustering of normal to medium sized mature megakaryocytes; 2 . normal to slight increased cellularity according to age; 3 . normal myeloid/erythroid ratio of bone marrow nucleated cells; [4] no increase of erythropoiesis; and 5. no increase of reticulin fibrosis ( $\mathrm{RF}$ grade 0 to 1 ). As compared to controls, the clustered megakaryocytes were more compact, of normal to increased size with slightly hyperlobulated nuclei (Figure 3), but less pronounced as compared to JAK2V617F mutated acquired ET and prodromal PV.

All affected members of the Dutch and Polish TPO HET family showed no spontaneous endogenous erythroid colony (EEC) formation in the absence of EPO and had normal EEC responses in the presence of EPO. Thrombopoietine (TPO) receptor/myeloproliferative leukemia (MPL) protein expression in platelets were decreased reflecting down regulated MPL ligand or TPO-receptor [9]. The decreased TPO-receptor/MPL expression was associated with increased MPL mRNA expression in platelets indicating an increased MPL receptor protein turn-over metabolism. From these clinical, laboratory and basic research studies it can be conscluded that increased levels of TPO in HET patients are indeed caused by a
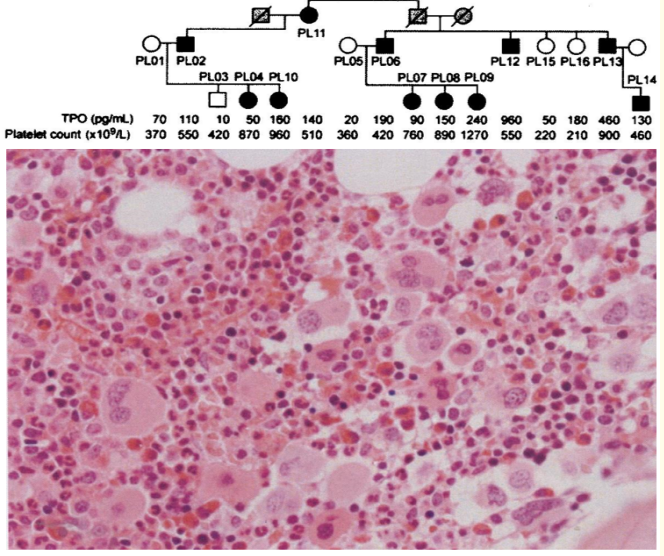

Figure 3: Pedigree of the Polish family with hereditary essential thrombocythemia (HET) due to germline gain of function mutation in the TPO gene (black) and related increased plasma TPO levels and platelet counts in affected ascompared to non-affected family members [8]. Bone marrow histology showed increase and loose to dense clustering of medium sized to large mature megakaryocytes, and normal myeloid to erythroid ratio of bone marrow nucleated cells, no to slight increased cellularity according to age, no increase of erythropoiesis, and no increase of reticulin fibrosis (RF grade 0 to 1 ). As compared to controls, the clustered mature normal and medium sized megakaryocytes were more compact with slightly hyperlobulated nuclei, but less pronounced as compared to acquired JAK2V617F mutated ET and prodromal polycythemia vera (PV, figures 5 and 6).

gain of function mutation in the TPO gene as the cause of increased plasma TPO levels that activates the normal TPO-receptor/MPL pathway thereby producing the typical ET phenotype of increased platelet counts and increase of polyclonal megakaryocytes arising from normal polyclonal hematopoietic hematopoietic stem cells.

\begin{tabular}{|c|c|c|c|c|c|c|}
\hline Case HET & Age & $\mathrm{Hb}$ & RBC & WBC & Platelets & TPO HET associated symptoms \\
\hline PL Family & Years & $\mathrm{g} / \mathrm{dL}$ & $\mathrm{x} 10^{12} / \mathrm{L}$ & $\mathrm{x} 10^{9} / \mathrm{L}$ & $\mathrm{x} 10^{9} / \mathrm{L}$ & \\
\hline PL11 & 84 & 15.0 & 5.0 & 10.6 & 550 & Acrocyanosis gangrene foot \\
\hline PL12 & $59 \mathrm{M}$ & 15.6 & 5.5 & 5.3 & $550-560$ & not available (na) \\
\hline PL13 & $58 \mathrm{M}$ & 15.0 & 5.0 & 7.7 & 510 & not available (na) \\
\hline PL06 & $56 \mathrm{M}$ & 14.5 & 5.0 & 6.5 & $408-410$ & None \\
\hline PL02 & $50 \quad \mathrm{~F}$ & 13.1 & 4.5 & 5.9 & $545-560$ & None \\
\hline PL04 & $30 \mathrm{~F}$ & 12.3 & 4.7 & 5.9 & $595-1300$ & headaches, hypertension \\
\hline PL07 & $28 \mathrm{~F}$ & 13.2 & 4.7 & 6.1 & $760-960$ & TIA, miscarriage erythromelalgia \\
\hline PL08 & $24 \mathrm{~F}$ & 13.5 & 4.7 & 7.1 & $750-890$ & erythromelalgia venous thrombosis \\
\hline PL09 & $24 \mathrm{~F}$ & 12.7 & 4.1 & 6.7 & $740-1340$ & Transient ischemic attacks (TIA) \\
\hline PL10 & $15 \mathrm{~F}$ & 14.2 & 4.1 & 10.6 & 960 & None \\
\hline PL14 & $14 \mathrm{M}$ & 12.3 & 4.6 & 6.2 & 460 & None \\
\hline
\end{tabular}

Table 1: Clinical and laboratory features in 11 affected members of the Polish (PL) family with autosomal dominant hereditary essential thrombocythemia (HET) caused by germline gain of function mutation in the TPO gene.

Citation: Jan Jacques Michiels., et al. "Analysis of Clinical, Laboratory and Bone Marrow Features in Dominant TPO, MPL and JAK2 Germline Mutated Hereditary Essential Thrombocythemia (HET) Versus Acquired MPL ${ }^{515}$, CALR and JAK2 ${ }^{\text {v617F }}$ Mutated ET in Myeloproliferative Neoplasms". Acta Scientific Medical Sciences 3.11 (2019): 86-96. 
$\mathrm{JAK2}^{\mathrm{V} 617 \mathrm{I}}$ and JAK2 ${ }^{\mathrm{R} 5640}$ mutated hereditary essential thrombocythemia: HET

Heterozygous JAK2 ${ }^{\mathrm{V} 6171}$ germline mutation has been desribed as the sole driver cause of ET phenotype in dominant JAK2 ${ }^{\mathrm{V} 617 \mathrm{I}}$-positive HET phenotype with increase of constitutively activated hypersensitive platelet and completely normal values for haemoglobin, haematocrit, erythrocytes, plasma TPO and serum EPO levels in six affected members of one family (Figure 4, Table 2) [14,15]. Case $\mathrm{C} 1$ presented at the age of 53 with MRI confirmed ischemic cerebral vascular events (CVE) at platelet count of $750 \times 10^{9} / \mathrm{L}$, which had been present for more than 10 years at levels between $700-970 \times 10^{9} /$ L. Sister C7 was diagnosed with myocardial infarction at platelet count between $338-536 \times 10^{9} / \mathrm{L}$. The children C4, C3 and C2 had persistent thrombocythemia and were on maintained low dose aspirin. Mother $\mathrm{C} 6$ was diagnosed with myocardial infarction at age 65 and developed ischemic CVE at the age of 72 and was subsequently anticoagulated with warfarin. Laboratory features of six affected members of the dominant JAK2 ${ }^{\mathrm{V} 617 \mathrm{I}}$ positive HET family revealed values for hemoglobin between 14.1 and 15.7 $\mathrm{g} / \mathrm{dL}$, red blood cells (RBC) between 4.6 and $4.9 \times 10^{12} / \mathrm{L}$, white blood cells (WBC) between 7.2 and $10.6 \times 10^{9} / \mathrm{L}$, platelet counts between 445 and $750 \times 10^{9} / \mathrm{L}$, and normal values for serum EPO, TPO and ferritine (Table 2) [14,15]. Bone marrow histology from case $\mathrm{C} 1$ demonstrated normal cellularity and architecture with increased numbers of clustered mature normal to medium sized megakaryocytes (Figure 4). Bone marrow histology from case C4 showed normal bone marrow cellularity and clustered increased numbers of normal to medium sized megakaryocytes (Figure 4) [15]. Staining for reticulin fibres in C1 and C4 showed no evidence of increased marrow fibrosis. Compared with controls, however, CFU-GM were increased and CFU-Mks were slightly increased in

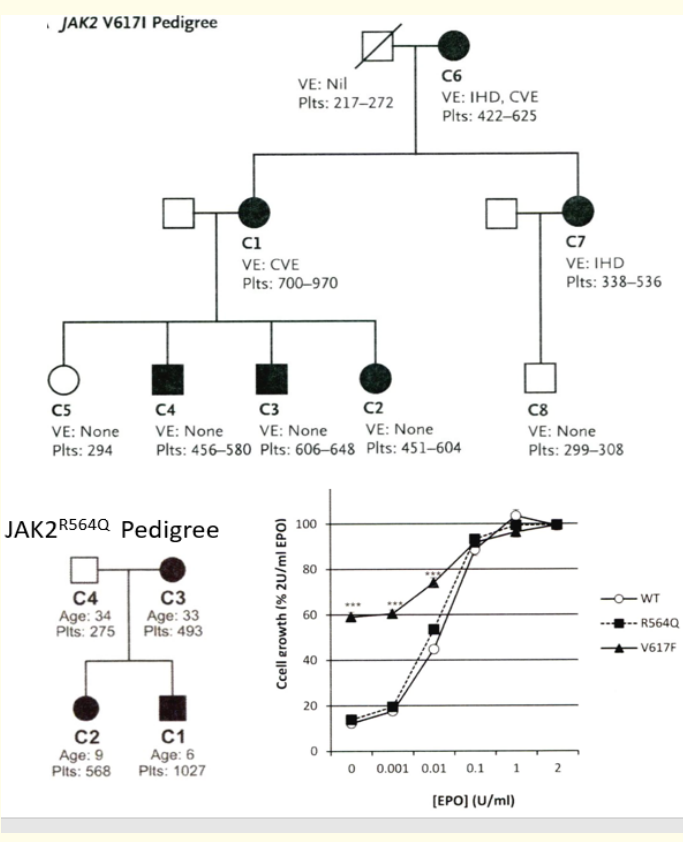

Figure 4: Upper. Pedigree of the UK family with hereditary essential thrombocythemia (HET) due to germline gain of function mutation JAK ${ }^{\mathrm{V} 6171}$ showing the presenting cerebral vascular events (CVE) IHD = ischemic heart disease and CVE = ischemic cerebrovascular event related to increased platelet counts in the affected JAK2 ${ }^{\text {V6171 }}$ mutated HET patients14,15.

Lower. Pedigree of the UK family with hereditary essntial thrombocythemia (HET) due to germline gain of function mutation JAK2 $2^{\mathrm{R} 540}$ as demonstrated by the need of EPO to induce EEC in JAK2 ${ }^{\mathrm{R} 564 \mathrm{Q}} \mathrm{HET} 16$ as compared to spontaneous EEC in acquired JAK2 ${ }^{\mathrm{V} 617 \mathrm{~F}} \mathrm{ET}$.

\begin{tabular}{|c|c|c|c|c|c|c|}
\hline JAK: & C1 & $\mathrm{C} 2$ & C3 & C4 & $\mathrm{C} 6$ & C7 \\
\hline Age at diagnosis & 53 & 34 & 36 & 38 & 79 & 61 \\
\hline Gender & $\mathrm{F}$ & $\mathrm{F}$ & M & M & $\mathrm{F}$ & $\mathrm{F}$ \\
\hline Hemoglobin g/dL & 15.7 & 14.7 & 15.6 & 14.1 & 15.4 & 14.9 \\
\hline $\mathrm{RBC} \times 10^{12} / \mathrm{L}$ & 4.6 & 4.7 & 4.9 & 4.9 & 4.6 & 4.7 \\
\hline MCV fl & 100 & 92 & 97 & 89 & 100 & 93 \\
\hline $\mathrm{WBC} \times 10^{9} / \mathrm{L}$ & 10.6 & 9.6 & 8.3 & 8.9 & 7.2 & 6.8 \\
\hline Neutrophils $\times 10^{9} / \mathrm{L}$ & 5.9 & 5.5 & 4.5 & 4.3 & 3.9 & 4.7 \\
\hline Platelets $\times 10^{9} / \mathrm{L}$ & 750 & 600 & 648 & 526 & 645 & 445 \\
\hline EPO IU/L & $\mathrm{nt}$ & 9.1 & 5.9 & 10.6 & $\mathrm{nt}$ & nt \\
\hline TPO pg/mL & $\mathrm{nt}$ & 99 & 113 & 79 & nt & nt \\
\hline Ferritin ug/L & $\mathrm{nt}$ & 127 & 146 & 290 & nt & nt \\
\hline \multicolumn{7}{|c|}{ V617I allelic level } \\
\hline MCN \% & 51 & 52 & 49 & 50 & 51 & 51 \\
\hline $\mathrm{CD} 66^{+} \%$ & 51 & 51 & 50 & 50 & nt & nt \\
\hline
\end{tabular}

Table 2: Laboratory characteristics of six affected members of dominant JAK2 ${ }^{\mathrm{V} 6171}$ positive Hereditary Essential Thrombocythemia (HET). Platelet counts in 6 affected family members ranged from 445 to $750 \times 10^{9} / \mathrm{L}$. 
Analysis of Clinical, Laboratory and Bone Marrow Features in Dominant TPO, MPL and JAK2 Germline Mutated Hereditary Essential Thrombocythemia (HET) Versus Acquired MPL ${ }^{515}$, CALR and JAK2 ${ }^{\mathrm{V} 617 \mathrm{~F}}$ Mutated ET in Myeloproliferative Neoplasms

the BM of JAK2 ${ }^{\mathrm{V} 6171}$-positive ET cases but BFUEs were not affected, thereby confirming the ET phenotype and lack of erythroid phenotype in JAK ${ }^{\mathrm{V} 617 \mathrm{I}}$ mutated HET [15]. Heterozygous germline JAK$2^{\mathrm{V} 617 \mathrm{I}}$ mutation induces sufficient cytokine hyperresponsiveness of the hematopoetic stem cells (HSC) to TPO but not for EPO as the mechanism to induce a homogeneous ET phenotype of increased platelet count in blood and megakaryocyte proliferation in the bone marrow without PV features [15].

Etheridge., et al. described a novel mutation JAK2 ${ }^{\mathrm{R} 564 \mathrm{Q}}$, identified in three affected asymptomatic family members with autosomal dominant hereditary essential thrombocythemia (HET) (Figure 4) [16]. JAK2 ${ }^{\mathrm{R} 564 \mathrm{Q}}$ and JAK2 ${ }^{\mathrm{V} 617 \mathrm{~F}}$ have similar levels of increased kinase activity by the demonstration that increased phosphorylation of JAK2 protein in platelets isolated from affected members of the family with the JAK2 ${ }^{\mathrm{V} 617 \mathrm{I}}$ and JAK2 ${ }^{\mathrm{R} 564 \mathrm{Q}}$ mutation as compared to a JAK2 wild type healthy controls [16]. In the absence of TPO, and at all concentrations of TPO, the growth charateristics of germline JAK2 ${ }^{\mathrm{R} 564 \mathrm{Q}}$-expressing hematopoietic progenitor cells in the bone marrow also showed significantly increased proliferation, compared to JAK2 wild type cells. Affected members of JAK2 ${ }^{\mathrm{R} 5640}$ HET showed normal EEC (Figure 4) and had normal values for plasma TPO and serum EPO thereby confirming that the heterozygous germline mutation JAK2 ${ }^{\mathrm{R} 564 \mathrm{Q}}$ is associated with ET phenotype without PV features [16].

MPL $^{\text {S505N }}$ germline mutated Hereditary Essential Thrombocythemia: HET

Teofili., et al. described the laboratory and clinical findings at time of observation and outcome of treatment in 21 affected family members (11 males, 10 females) from 7 HET families carrying the dominant MPL ${ }^{\mathrm{S} 505 \mathrm{~N}}$ mutation [17]. Mild to moderate splenomegaly was detected in 9 of 20 cases. Ten children or adolescents at ages between 1 and 18 year presented with high platelet counts between 626 to $1553 \times 10^{9} / \mathrm{L}$ and no splenomegaly or bone marrow fibrosis (except minir splenomegaly in 2) during follow-up periods of 2 to 24 years [17]. Two of 11 cases were asymptomatic except increased platelet count and nine of 11 cases at ages between 23 and 80 years presented with bone marrow fibrosis and splenomegaly (spleen sizes on echogram 15 to $19 \mathrm{~cm}$ ) at hemoglobin levels between 10.1 and $15.5 \mathrm{~g} / \mathrm{dL}$, platelet counts between 408 and $1210 \times 10^{9} / \mathrm{L}$ and normal leuokocytes. Low dose aspirin was given in 12 of $21 \mathrm{MPL}^{\mathrm{S} 505 \mathrm{~N}} \mathrm{HET}$ patients as prevention or symptomatic relief of microvascular disturbances. Four of 21 affected family members experienced major thrombosis including fatal stroke in 2 at the age of 76 and 80, myocardial infarction at age 31 and transient ischemic attack at age 41 . The bone marrow in three affected adolescent MPL patients was slightly hypercellular and show increased of and atypical large megakaryocytes with nuclear deviations and reticulin fibrosis was absent [17]. The bone marrow in two cases at age of 43 and 69 showed increase of dense clustered atypical large megakaryocytes and loose network of reticulin with many intersection [17]. The bone marrow in some of the affected adult or elderly patients was characterized by a hypocellular bone marrow with diffuse increase of reticulin fibrosis grade 3 (RF 3) [17].

Acquired JAK2 ${ }^{\mathrm{V} 617 \mathrm{~F}}$ mutated ET, prodromal PV and classical PV

Between 1975 and 2015 Michiels., et al. produced novel diagnostic criteria for ET and PV by including bone marrow histology according to the Rotterdam Clinical and Pathological (1980 RCP) [6,7], the European Clinical and Pathological (2002 ECP) [10] and the European Clinical, Molecular, and Pathological (2006-2015 ECMP) [11-13]. criteria for prefibrotic ET and PV and primary chronic megakaryocytic granulocytic myeloproliferation (PMGM). Red cell count at a cut-off level of $6 \times 10^{12} / \mathrm{L}$ separates JAK2 ${ }^{\mathrm{V} 617 \mathrm{~F}} \mathrm{mu}-$ tated ET from PV and obviates the need for red cell mass measurement when bone marrow histology. JAK2 ${ }^{\mathrm{V} 617 \mathrm{~F}}$ mutation screening is the first step in the diagnostic work-up of MPNs [12]. In JAK2 ${ }^{\mathrm{v} 617 \mathrm{~F}}$ trilinear MPN mutated ET and PV patients the pleomorphic medium to large megakaryocytes in bone marrow smears and bone marrow biopsy were comparable regarding size and degree of pleomorphy (Figures 6 and 7) [13]. The prefibrotic stages of JAK2 ${ }^{\mathrm{V} 617 \mathrm{~F}}$ ET can easily be diagnosed clinically without use of bone marrow biopsy histology. We prospectively evaluated the ECMP criteria for the diagnosis of myeloproliferative neoplasms (MPNs) in 6 JAK2 ${ }^{\mathrm{V} 617 \mathrm{~F}}$ mutated ET and 4 PV patients during long-term follow-up in view of critical analysis of the literature [13]. Bone marrow histology in acquired JAK2 ${ }^{\mathrm{V} 617 \mathrm{~F}}$ mutated ET complicated by erythromelalgia is typically features by increase of clustered large mature pleomorphic megakaryocytes with normal $(<60 \%)$ to increased celllarity (60 - 80\%) due to increased erythropoiesis [13]. ET with increased erythropoiesis in the bone marrow and decreased serum EPO levels mimicking PV is not seen in the TPO-mutated Dutch and Polish HET families [9] and also not in JAK2 ${ }^{\mathrm{V} 6171}$ mutated HET showing increase of clustered large megakaryocytes in a normocellular bone (Figures 6 and 7) [14-16]. There is local increase of erythropoiesis in areas of loose clustered pleiomorphic megakaryoctyes in normocelluar JAK2 ${ }^{\mathrm{V} 617 \mathrm{~F}}$ mutated ET, prodromal PV and early stage PV, which is not seen in MPL ${ }^{515}$ mutated ET and CALR mutated ET [1720]. Patients with JAK2 ${ }^{\mathrm{V} 617 \mathrm{~F}}$ positive ET mimicking PV show spontaneous endogenous erythroid colony formation (EEC) and have low serum EPO levels [11-13]. EEC and srum EPO levels are normal in MPL [18] and CALR [19,20] mutated ET, and in congenital TPO and JAK2 ${ }^{\mathrm{V} 617 \mathrm{I}}$ mutated HET patients (Table 3) $[9,14,15]$. Rumi., et al. found a novel germline JAK2 ${ }^{\mathrm{H} 608 \mathrm{~N}}$ mutation as the cause of HET [21] using next generation sequencing (NGS) in 61 cases with familial clustering of MPN [22]. Three heterozygous JAK2 ${ }^{\mathrm{H} 608 \mathrm{~N}}$ mutated HET patients father, sun and grandchild showed isolated thrombocythemia with platelet count of 600,648 and $342 \times 10^{9} / \mathrm{L}$ (normal values $155-295 \times 10^{9} / \mathrm{L}$ in healthy individuals with normal C-reactive proteon: CRP). Bone marrow histology revealed increase of large 
megakaryocytes in a normocellular bone marrow (Rumi, personal communication), without splenomegaly and bone marrow fibrosis.

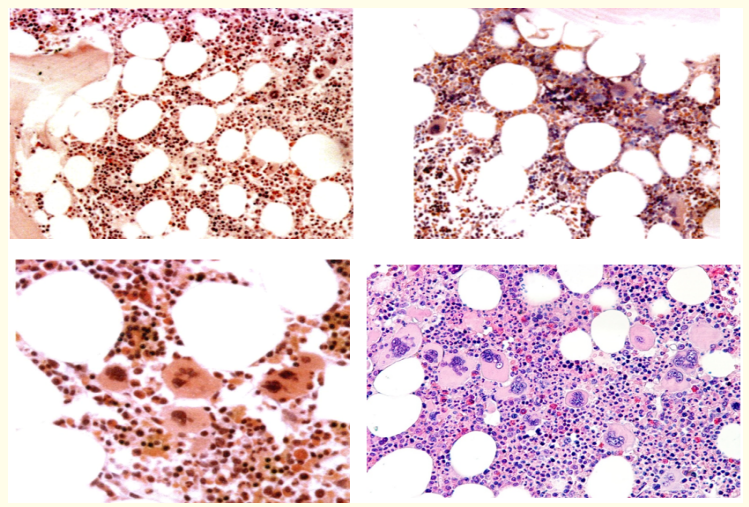

Figure 5: Upper lef and right, lower left. Bone marrow histology from case C1 (left panels) and from case C4 of the family with JAK2 ${ }^{\mathrm{V} 6171}$ mutated HET showing a normocellular bone marrow with increase and clustering of large megakaryocytes with some lobulation of the nuclei consistent with ET. Lower right. JAK2 ${ }^{\mathrm{V} 617 \mathrm{~F}}$ positive ET showing hypercellular bone marrow due to erythroid hyperplasia (65\%) and marked hyperplasia of large pleomorphic megakaryocytes with hyperlobulated nuclei mimicking PV (prodromal PV) in a 65 years old man with erythrocytes $5.4 \times 10^{12} / \mathrm{L}$, hemoglobin $15.8 \mathrm{~g} / \mathrm{dL}, \mathrm{MCV} 89$, leukocytes $12 \times 10^{9} / \mathrm{L}$, platelets $517 \times 10^{9} / \mathrm{L}$, LDH $600 \mathrm{UI} / \mathrm{L}$ (JAK2 ${ }^{\mathrm{V} 617 \mathrm{~F}}$ mutation allele burden: 20\% on peripheral blood granulocytes). No reticulin fibrosis.
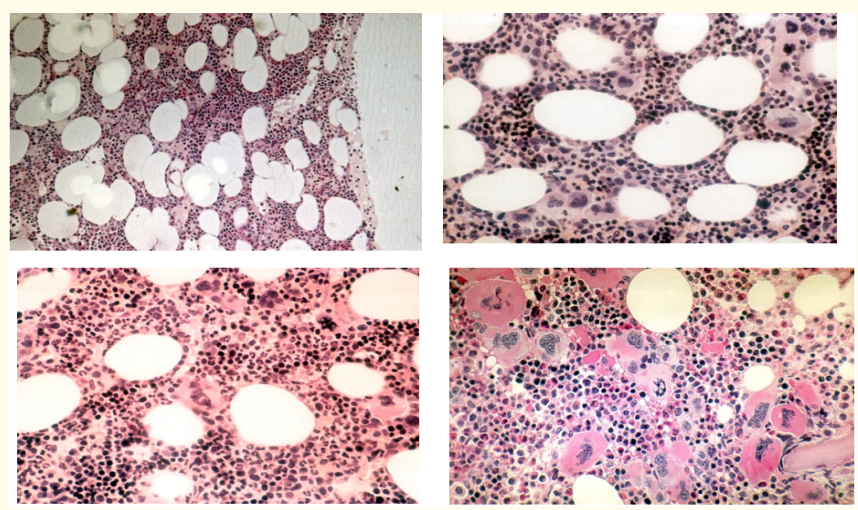

Figure 6: Typical ET (upper), ET/PV (lower left), and PV (lower right) bone marrow features in ET and PV patients showing increase of large mature megakaryocytes in a normocellular or increased cellularity duet o increased erythropiesis in prodromal PV and classical PV. Pleiomorphic megakaryocytes in ET (upper panels) have less hyperlobulated nuclei as compared to PV (left bottum). The LAP score is increased in ET and PV complicated by erythromelalgia and the clustered pleiomorphic megakaryocytes in prefibrotic ET ET/PV and PV patients complicated by erythromelalgia are identical. Dr Lam, Pathology Laboratory, Erasmus University Rotterdam and Dr De Raeve Pathology Laboratory Brussels University Hospital.
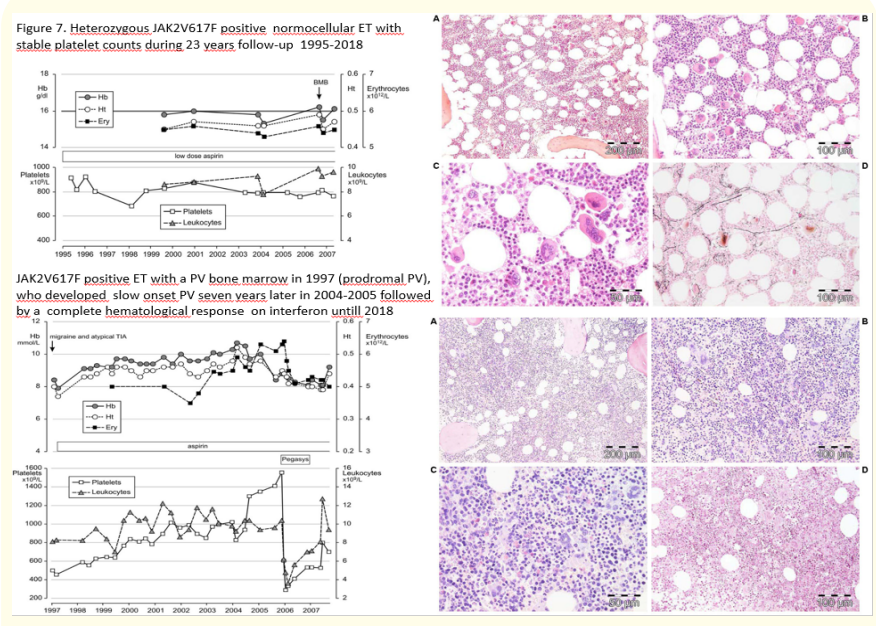

Figure 7: Upper figure and bone marrow pictures. Heterozygous JAK2V617F positive normocellular ET with stable platelet counts during 23 years follow-up 1995-2018. Lower figure and bone marrow pictures. JAK2 ${ }^{\mathrm{V} 617 \mathrm{~F}}$ positive ET with a PV bone marrow in 1997 (prodromal PV), who developed slow onset PV seven years later in 2004-2005 followed by a complete hematological response on interferon untill 2018. Observations by Drs Michiels and De Raeve.

\section{Acquired MPL ${ }^{515}$ mutated thrombocythemia}

Bone marrow histology from a patient with thrombocythemia carrying the MPLW ${ }^{515 L}$ mutation displayed clusters of large megakaryocytes with a greater number of giant megakaryocytes with hyperlobulated stag-horn nuclei in a normal cellular bone marrow and no increase of erythropoiesis (Figure 8) [18]. The presence of clustered small and giant megakaryocytes with deeply lobulated staghorn like hyperlobulated nuclei in MPL ${ }^{515}$ mutated thrombocythemia (Figure 8), which are not seen in JAK2 ${ }^{\mathrm{V} 617 \mathrm{~F}}$ mutated ET and PV (Figures 6 and 7) and CALR thrombocythemia (Figures 9 and10). Increase of erythropoiesis is not seen in MPL ${ }^{515}$ mutated ET (Figure 8) [18]. MPL ${ }^{515}$ mutated ET have no clinical, laboratory and bone marrow features of prodromal PV at diagnosis, do not evolve into PV during follow-up [18]. MPL ${ }^{515}$ mutated normocellular ET have normal values for LAP score, serum EPO and ferritin levels [18]. JAK2 ${ }^{\mathrm{V} 617 \mathrm{~F}}$ mutated ET show local increase of erythropoiesis in areas of loose clustered pleiomorphic megakaryocytes in normocelluar JAK2 ${ }^{\mathrm{V} 617 \mathrm{~F}}$ mutated ET, whereas bone marrow is hypercellular due to increased erythropoiesis and megakaryopoiesis (EM) $[12,13]$ JAK2 ${ }^{\mathrm{V} 617 \mathrm{~F}}$ mutated prodromal PV and classical PV have increased score for leukocyte alkaline phosphatase (LAP) stain, low serum EPO and pleomorphic medium sized to large mature megakaryocyte morphology. The natural history of MPL ${ }^{515}$ normocellular ET is best reflected by decreased cellularity due to decreased erythropoiesis and increase of reticulin fibrosis (RF) from grade 0 to grade 1,2 and 3 [18]. 

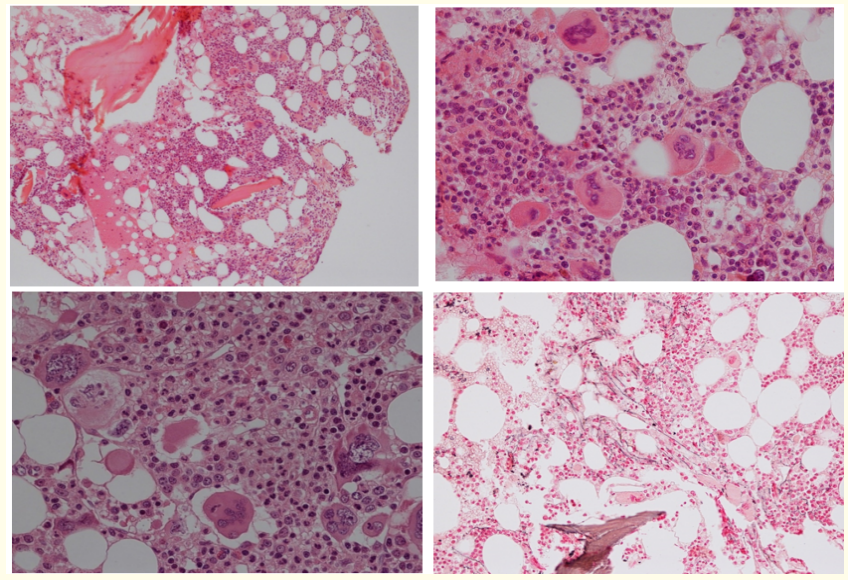

Figure 8: Bone marrow histology of MPL ${ }^{515}$ mutated ET (asymptomatic woman, age 78 year with platelet count of $1379 \times 10^{9} / \mathrm{L}$ as the only abnormality) showing increase and clustering of large and giant mature megakaryocyteswith hyperlobulated staghornlike hyperlobulated nuclei in a normocellular bone marrow with increased reticulin fibrosis grade 2 (RF 2)18. Observations Dr De Raeve University Hospital Brussels.

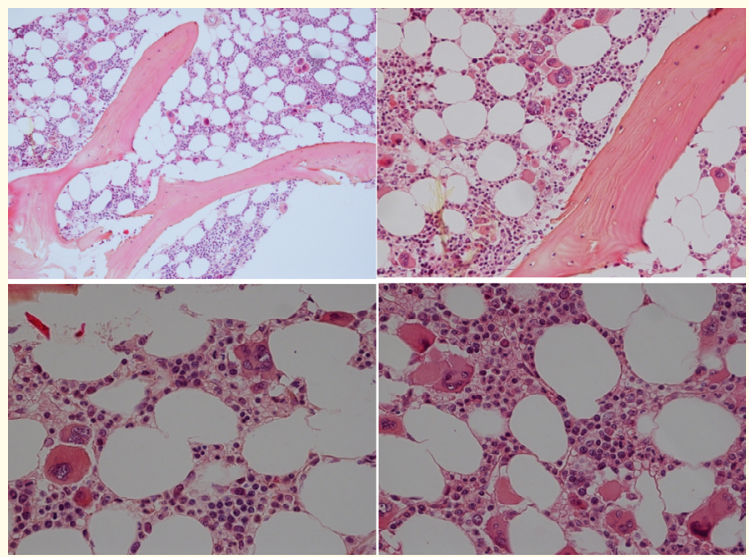

Figure 9: Bone marrow histology of CALR mutated ET/PMGM case 6 (female, age 63 year, table 7) showing dense clusters of large immature megakaryocytes with dysmature cloud-like nuclei in a normocellular MGM bone marrow with relative reduction of erythropoiesis and no increase of reticuline fibrosis grade $0(\mathrm{RF} 0)^{19,20}$.

Observations Dr Potters, Valster, Michiels, and De Raeve.

\section{Acquired CALR mutated thrombocythemia}

Michiels and De Raeve recently described 13 consecutive cases of CALR thrombocythemia previously diagnosed as JAK2/MPL wild type prefibrotic ET associated with a typical PMGM bone marrow histology (ET/PMGM (Figures 9 and 10) in 11 cases (85\%) and with MF/PMGM in two cases (15\%) [19]. Two ET/PMGM cases presented with fatigue only. All $11 \mathrm{ET} / \mathrm{PMGM}$ and the $2 \mathrm{MF} /$ PMGM were relative asymptomatic and not suffering from consti-

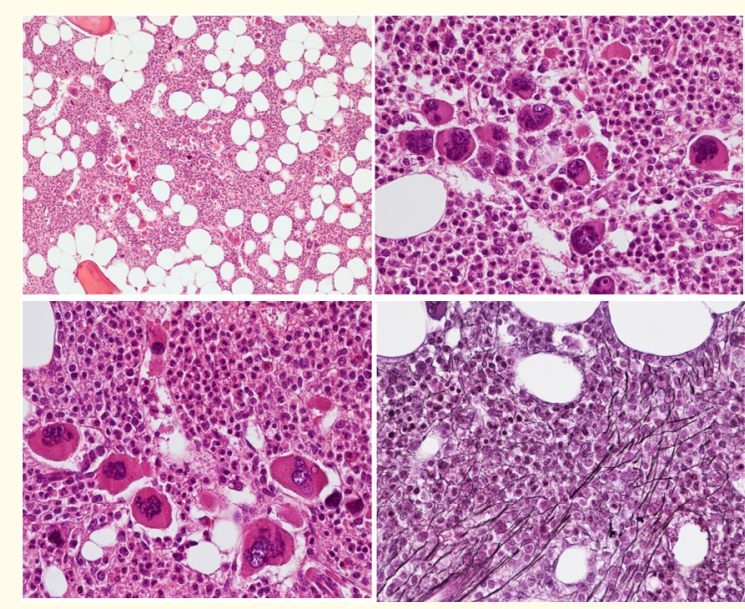

Figure 10: Bone marrowhistology of CALR mutated ET/PM case 9 (man age 73 year, table 7) showing loosely and dense clusters of large megakaryocytes with dysmature, bulky, cloud-like nuclei in a normocellular to slightly increased cellular bone marrow with increase of reticulin fibers (RF 2)19,20. Observations Dr Potters, Valster, Michiels and De Raeve.

tuional symptoms. Platelet counts in ET/PMGM ranged from 536 to $1306 \times 10^{9} / \mathrm{L}$ at time of first presentation and the two MF/PMGM cases had platelet counts of 265 and $347 \times 10^{9} / \mathrm{L}$ respectively [19]. The values for hemoglobin, erythrocytes and white blood cells were in the normal range before and after follow-up in all cases of ET/PMGM. Bone marrow histology in prefibrotic CALR Thrombocythemia show dysmorphic megakaryocytes with definite abnormalities of maturation with bulky (bulbous) hyperchromatic nuclei and some disturbances of the nuclear cytoplasmic ratio consistent with CALR mutated PMGM (Figures 9 and 10), which are not seen in MPL ${ }^{515}$ mutated ET (Figure 8) and also not in JAK2 ${ }^{\mathrm{V} 617 \mathrm{~F}}$ mutated ET, prodromal PV and classical PV (Figures 6 and 7). The natural history of CALR thrombocythemia and myelofibrosis is best reflected by the degree of anemia, splenomegaly, bone marrow cellularity due to dual megakaryocytic granulocytic myeloproliferation (CALR MGM) and increase of reticulin fibrosis [19,20].

Comparative analysis in table 3 showed that JAK2 ${ }^{\mathrm{V} 6711}$ HET patients and acquired JAK2 ${ }^{\mathrm{V} 617 \mathrm{~F}}$ ET and PV patients are symptomatic presenting erythromelalgic microvascular manifestations already at platelet counts between 400 and $1000 \times 10^{9} / \mathrm{L}$ labeled by Michiels between 1985 and 2017 as JAK2 ${ }^{\mathrm{V} 617 \mathrm{~F}}$ mutated erythromelalgic thrombotic thrombocythemia: ETT, platelet microvascular thrombophilia (PMT) or Sticky Platelet Syndrome (SPS) [6,23-25]. TPO mutated HET and acquired MPL ${ }^{515}$ ET patients also present with aspirin responsive ETT at platelet count between 627 to $1726 \times 10^{9} / \mathrm{L}$. Erythromelalgic thrombotic thrombocythemia (ETT) was not recorded in CALR mutated ET/PMGM at platelet counts between 400 and 1000x10/L (CALR thrombocythemia). Leukocyte alkaline phaspatase stain is typically increased in acquired JAK2 $2^{\mathrm{V} 617 \mathrm{~F}}$ mutat- 
Analysis of Clinical, Laboratory and Bone Marrow Features in Dominant TPO, MPL and JAK2 Germline Mutated Hereditary Essential

Thrombocythemia (HET) Versus Acquired MPL ${ }^{515}$, CALR and JAK2 ${ }^{\text {V617F }}$ Mutated ET in Myeloproliferative Neoplasms

ed ET similar as in JAK2 ${ }^{\mathrm{V} 617 \mathrm{~F}}$ prodromal PV and classical PV (Table 3). In contrast, low to decreased LAP scores has been reported recently in CALR mutated ET/PMGM cases. CALR thrombocythemia typically shows prefibrotic PMGM bone marrow histology featured by dysmorphic immature megakaryocytes with cloud-like nucle, which are never seen in JAK2 ${ }^{\mathrm{V} 617 \mathrm{~F}}$ mutated ET and PV and also not in acquired MPL ${ }^{515}$ mutated thrombocythemia [18-20].

\section{Discussion}

With the advent of TPO, MPL, JAK2 and CALR mutations as driver causes of congenital or acquired thrombocythemia the PVSG-WHO criteria have lost their significance for the classifications of the myeloproliferative neoplasms into ET, PV and PMF (Figure 11) anno 2018-2020 [26-31]. ET is not essential anymore and consists of at

\begin{tabular}{|c|c|c|c|c|c|c|}
\hline Category HET vs ET & Number patients & Platelet & $9 /$ L Range & Plasma TPO pg/mL & EEC/ EPO -/+ & LAP score \\
\hline Dutch TPO HET & 10 & 880 & 1280 & Increased & $\operatorname{Neg}(-)$ & *Normal \\
\hline Polish TPO HET & 11 & 701 & 1340 & Increased & $\operatorname{Neg}(-)$ & Normal \\
\hline JAK2 ${ }^{\mathrm{V} 6171}$ HET & 6 & 445 & 750 & Normal & $\operatorname{Neg}(-)$ & Normal \\
\hline JAK2 $2^{\mathrm{V} 617 \mathrm{~F}}$ ET & 6 & 425 & 814 & Decreased & Pos $(+)$ & Increased \\
\hline MPL $^{5505 N}$ HET & & & & Normal & $\operatorname{Neg}(-)$ & Normal \\
\hline MPL ${ }^{\text {w515L/K }} \mathrm{ET}$ & 23 & 380 & 1500 & Normal & Neg $(-)$ & Normal \\
\hline Calr Et & 10 & 714 & 1306 & Normal & $\operatorname{Neg}(-)$ & Decreased \\
\hline
\end{tabular}

Table 3: Characteristic findings in TPO, JAK2 ${ }^{\mathrm{V} 6171}$ and MPL ${ }^{\mathrm{S505N}}$ hereditary essential thrombocythemia (HET) versus JAK2 ${ }^{\mathrm{V} 617 \mathrm{~F}}, \mathrm{MPL}^{515}$ and CALR mutated acquired essential thrombocythemia (ET)

*Predicted to be normal

least four distinct entities of congenital TPO, JAK2 or MPL mutated hereditary thrombocythemia (HT) and acquired MPL and CALR mutated thrombocythemias, which mutally exclude each other. Primary myelofibrosis (PMF) is not a disease, but a secondary event of reactive reticuline fibrosis in all variants of thrombocythemia caused by congenital germline or acquired somatic mutations in the TPO, MPL, JAK2 and CALR genes [32]. The PVSG-WHO did not considered dominant hereditary ET as part of the classification of the MPNs. The PVSG-WHO did not use increased erythrocytes above $6 \times 10^{12} / \mathrm{L}$ asa key feature of trilinear PV proposed by Dameshek [33,34]. The PVSG-WHO did not use bone marrow histology to as pathogomonic clues to distinguish JAK2 ${ }^{\mathrm{V} 617 \mathrm{~F}}$ ET mimicking PV from and CALR and MPL mutated ET without features of PV as distinct MPNs at the clinical, laboratory and bone marrow level [18-20]. The natural history of each of these JAK2 ${ }^{\mathrm{V} 617 \mathrm{~F}} \mathrm{mu}-$ tated masked PV, and MPL and CALR is feaured by anemia, splenomegaly and increased of bone marrow reticuln fibrosis grade 01,2 , 3 and 4 in the ECMP classifications 2006 to 2020. The PVSG-WHO have overlooked and misinterpreted the original description and clinical laboratory and bone marrow definition of $\mathrm{PV}$ as a trilinear MPD by Dameshek (1950) [33,34], preceded by ET as discovered by Michiels between 1975 and 1985 to clearly distinct ET and PV patients according o the RCP and ECP criteria [10-13,35,36]. Georggi and Michiels worked together between 1987 and 1998 and defined prefibrotic hypercellular ET associated with Essential or Primary Megakaryocytic, Granulocytic Myeloproliferation (PMGM) as the third distinct MPD entity without features of PV or normocellular ET at the clinical laboratory and bone marrow level [10-13]. In retrospect, the defintion of megakaryocytic leukemia
ML by Dameshek (1951) [37] has been classified as PTH with a minimum platelet count of $1000 \times 10^{9} / \mathrm{L}$ by the PVSG in 1975 and by Thiele., et al. in 1988 (Figure 12) [36-40]. The diagnostic clinical laboratory and bone marrow differential diagnostic criteria of PV

Changing
face
of Acquired ET

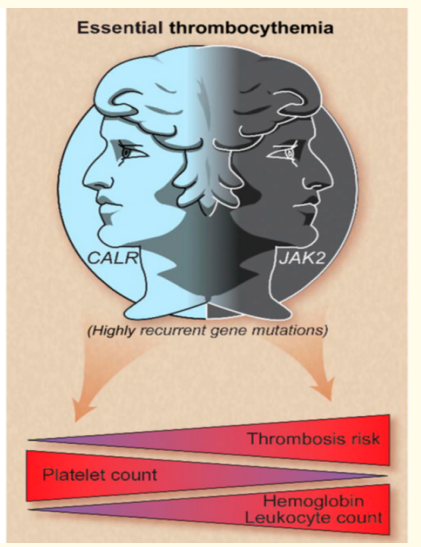

Figure 11: PVSG-WHO defined essential thrombocythemia (ET) is is not essential anymore and consists of at least four distinct entities of congenital JAK2, MPL and TPO mutated hereditary thrombocythemia (HT) and three distinct acquired JAK2 ${ }^{\mathrm{V} 617 \mathrm{~F}}$, MPL ${ }^{515}$ and CALR Thrombocythemia, which mutally exclude each other. JAK2 ${ }^{\mathrm{V} 617 \mathrm{~F}}$ mutated Thrombocythemia patients reveal spontaneous endogenous erythroid colony formation (EEC), increased LAP score and low serum EPO levels consistent with prodromal PV. EEC, LAP score and serum EPO levels are normal in MPL ${ }^{515}$ and CALR mutated Thrombocythemia and in TPO and JAK2 germline mutated HET patients 
and PTH at that time (Figure 12) clearly show that PTH has charecteristic features of JAK2 wild type ET caused by CALR or MPL somatic mutations without any features of PV as defined by ECMP classification 2014-2020 in the studies of Michiels and De Raeve

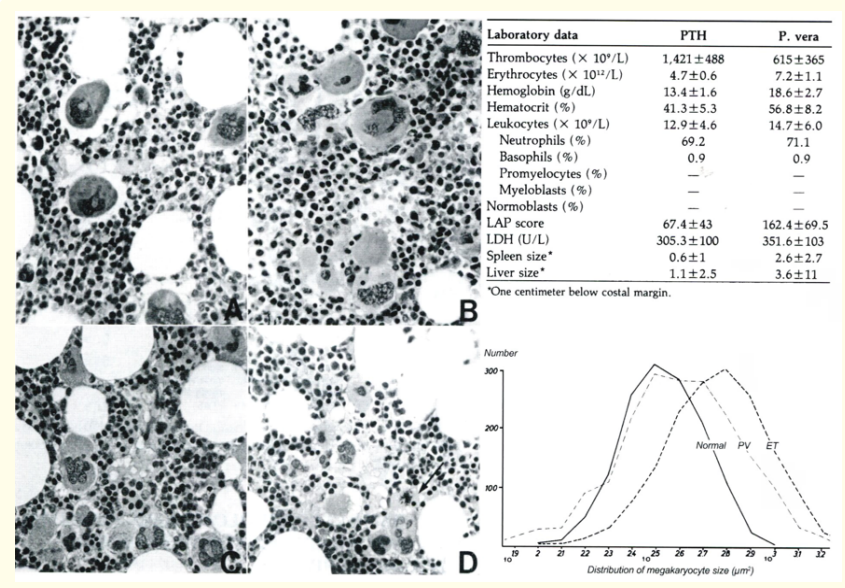

Figure 12: Histopathology of PVSG (1975) defined primary thrombohemorrhagic (PTH) or essential thrombocythemia (ET) showing normal values of erythrocytes, LAP score below 100 and no or minor splenomegaly versus polycythemia vera (PV) featured by increased erythrocytes above $6 \times 10^{9} / \mathrm{L}$, increased LAP score and minor to moderate splenomegaly. Bone marrow biopsy histology of PVSG (1985) defined PTH at platelet counts above $1000 \times 10^{9} / \mathrm{L}$ PTH is characterized by increase of large to giant megakaryocytes disperesed among normal granulopoiesis and erythropoiesis $(\mathrm{A}, \mathrm{B})$. Bone marrow biopsy histology in P.vera is characerized by marked increase of clustered pleomorphic medium sized megakaryotes with increase of erythropoiesis (C,D)36-40.

\section{Conclusion}

The spectrum of aspirin responsive sticky platelet mediated thrombophilia (SPT) in hereditary essential thrombocythemia (HET) due to germline gain of function mutations in the TPO, JAK2 and MPL genes is comparable to the spectrum of SPT in acquired essential thrombocythemia (ET) caused by somatic gain of function mutations JAK2 ${ }^{\mathrm{V} 617 \mathrm{~F}}$ and MPL ${ }^{515}$. Increase of large platelets in blood smears and large mature megakaryocytes with hyperploid nuclei in a normal cellular bone marrow were diagnostic for autosomal dominant HET and for acquired ET. Evolution of HET and ET into secondary myelofibrosis (MF) belong to the natural history of TPO, JAK2, MPL mutated HET and acquired mutated JAK2 ${ }^{\mathrm{V} 617 \mathrm{~F}}$ and MPL ${ }^{515}$ mutated acquired ET. In TPO and the JAK2 gene JAK$2^{\mathrm{V} 6171}$ and JAK2 ${ }^{\mathrm{R} 564 \mathrm{Q}}$ mutated HET the responses of mutated CD33 and CD34 + cells to TPO are increased, but the responses to EPO were normal thereby explaining why HET caused by heterozygous germline TPO and JAK2 mutations are associated with the biological characteristics of ET without PV features. Acquired MPL ${ }^{515}$ and CALR mutated ET has no PV features whereas acquired JAK2 ${ }^{\mathrm{V} 617 \mathrm{~F}}$
ET is associated with typical features of PV in blood and bone marrow including low serum EPO and spontanuous endogenous erythroid colony (EEC) formation. CALR mutated ET and BCR/ABL positive ET are associated with the production of indolent platelet with the absence of sticky platelet mediated thrombophilia and show a rather high tendency of ET evolution into myelofibrosis.

\section{Contribitions of Authors}

JJM and HDR designed the study and wrote the manuscript. FTK, $\mathrm{KL}, \mathrm{AP}, \mathrm{VP}$ and FV significant contributed to data and scientific content.

\section{Bibliography}

1. Villeval JL., et al. "High thrombopoietin production by hematopoietic cells induces a fatal myeloproliferative syndrome in mice". Blood 90 (1997): 4369-4383.

2. Yan XD., et al. "A model of myelofibrosis and osteosclerosis in mice induced by overexpressing thrombopoietin (mpl ligand); reversal of disease by bone marrow transplantation". Blood 88 (1996): 402-409.

3. Vannucchi A., et al. "A pathobiologic pathway linking thrombopoietin GATA-1, and TGF-beta1 in the development of myelofibrosis". Blood 105 (2005): 3493-3501.

4. Schlemper RJ., et al. "Familial essential thrombocythemia: clinical charateristics of 11 cases in one family". Annals of Hematology 68 (1994): 153-158.

5. Wiestner A., et al. "An activating splice donor mutation in the thrombopoietin gene causes hereditary thrombocythemia". Nature Genetics 18 (1998): 49-52.

6. Michiels JJ., et al. "Erythromelalgia caused by platelet mediated arteriolar inflammation andthrombosis in thrombocythemia". Annals of Internal Medicine 102 (1985): 466-471.

7. Michiels JJ and Juvonen E. "Proposal for revised diagnostic criteria of essential thrombocythemia and polycythememia vera by the Thrombocythemia Vera study Group (TVSG)". Seminars in Thrombosis and Hemostasis 23 (1997): 339-347.

8. Liu K., et al. "A de novo splice donor mutation in the thrombopoietin gene causes hereditary thrombocythemia in a Polish family". Haematologica 93.5 (2008): 706-714.

9. Kralovics R., et al. "Comparison of molecular markers in a cohort of patients with chronic myeloproliferative disorders". Blood (2003): 1869-1871.

10. Michiels JJ and Thiele J. "Clinical and pathological criteria for the diagnosis of essential thrombocythemia, polycythemia vera and idiopathic myelofibrosis (agnogenic myeloid metaplasia)". International Journal of Hematology 76 (2002): 133145. 
11. Michiels JJ., et al. "Changing concepts of diagnostic criteria of myeloproliferative disorders and the molecular etiology and classification of myeloproliferative neoplasms: From Dameshek 1950 to Vainchenker 2005 and beyond". Acta Haematologica 133 (2015): 71-86.

12. Michiels JJ., et al. "Increased erythrocyte count on top of bone marrow histology, but not by EPO level or JAK2V617F mutation load discriminates between JAK2V617F mutated essential thrombocythemia and polycythemia vera". Journal of Hematology and Thromboembolic Diseases (2015).

13. Michiels JJ., et al. "The European clinical, Molecular and Pathological (ECMP) criteria and the 2007/2008 revision of the World Health Organization for the diagnosis, classfication and staging of prefibrotic myeloproliferative neoplasms carrying the JAK2V617F mutation". Turkish Journal of Hematology 31 (2014): 239-254.

14. Mead AJ., et al. "Germline JAK2 mutation in a family with hereditary thrombocytosis". The New England Journal of Medicine 366 (2012): 967-969.

15. Mead AJ., et al. "Impact of isolated germline JAK2V617I mutation on human hematopoiesis". Blood 121 (2013): 4156-4165.

16. Etheridge SL., et al. "A novel activating, germline JAK2 mutation, JAK2R564Q, causes familial essential thrombocytosis". Blood 123 (2014): 1059-1068.

17. Teofili L., et al. "Hereditary thrombocytosis caused by MPLSer505Asn is associated with a high thrombotic risk, splenomegaly and progression to bone marrow fibrosis". Haematologica 95 (2010): 65-70.

18. Michiels JJ., et al. "Bone marrow characteristics in MPL515 mutated thrombocythemia with various degress of myelofibrosis: A cross sectional follow-up study in eight cases". Journal of Hematology and Thromboembolic Diseases 6 (2018): 2.

19. Michiels JJ., et al. "Bone marrow histology in CALR mutated thrombocythemia and myelofibrosis: results fro two cross sectional studies in 70 newly diagnosed JAK2/MPL wild type thrombocythemia patients". International Journal of Bone Marrow Research 2 (2019): 064-078.

20. De Raeve H., et al. "Novel Clinical, Laboratory, Molecular and Pathological (2018 CLMP) Criteria for the Differential Diagnosis of three Distinct JAK2, CALR and MPL Mutated Myeloproliferative Neoplasms: The Role of Driver Mutation Analysis and Bone Marrow Histology". International Journal of Cancer Research and Therapy 3.2 (2018):1-12.

21. Rumi E., et al. "A novel germline JAK2 mutation in familial myeloproliferative neoplasms". American Journal of Hematology 89.1 (2014):117-117.
22. Rumi E., et al. "Familial chronic myeloproliferative disorders: Clinical phenotype and evidence of disease anticipation". Journal of Clinical Oncology 25 (2007): 5630-5635.

23. Michiels JJ., et al. "Aspirin responsive platelet thrombophilia in essential thrombocythemia and polycythemia vera". World Journal of Hematology 2.2 (2013): 20-43.

24. Michiels JJ., et al. "Platelet-mediated erythromelalgic, cerebral, ocular, and coronary microvasculae ischemic and thrombotic manifestations in patients with essential thrombocythemia and polycythemia vera: a distinct aspirin-responsive and coumadin-resistent arterial thrombophilia". Platelets 17.8 (2006): 528-544.

25. Michiels JJ. "Aspirin cures erythromelalgia and cerebrovascular distubances in JAK2-thrombocythemia". World Journal of Hematology 6.3 (2017): 32-54.

26. Chao MP and Gotlib J. "Two faces of ET: CALR and JAK2". Blood 123.10 (2014): 1438-1439.

27. Klampf T., et al. "Somatic mutations od calreticulin in myeloproliferative neoplasms". The New England Journal of Medicine 369 (2013): 2379-2387.

28. Nangalia J., et al. "Effect of mutation order on myeloproliferative neoplasms". The New England Journal of Medicine 372.7 (2015): 601-612.

29. Rotunno G., et al. "Impact of calreticulin mutations on clinical and haematological phenotype and outcome in essential thrombocthemia". Blood 123.10 (2014):1552-1555.

30. Rumi E., et al. "JAK2 or CALR mutation status defines subtypes of essential thrombocythemia with substantially different clinical course and outcome". Blood 123 (2014): 1552-1515.

31. Grinfeld J., et al. "Classification and Personalized Prognosis in Myeloproliferative Neoplasms". The New England Journal of Medicine 379 (2018): 1416-1430.

32. Michiels JJ., et al. "Myelofibrosis is a Secondary Event in JAK2 Trilinear Myeloproliferative Neoplasm (MPN) and in CALR and MPL Thrombocythemia: Implications for Novel Treatment Options of Prefibrotic MPN". Journal of Hematology and Thromboembolic Diseases 5 (2017b): 5.

33. Dameshek W. "Physiopathology and corse of polycythemia vera as related to therapy". Journal of the American Medical Association 142 (1950): 790-797.

34. Michiels JJ. "Physiopathology, etiologic factors, diagnosis and course of polycythemia vera as related to therapy according to William Dameshek 1940-1950". Turkish Journal of Haematology 30 (2013a): 102-110. 
35. Thiele J., et al. "Primary (essential) thrombocythemia versus polycythemia rubra vera. A histomorphometric analysis of bone marrow features in trephine biopsies". Analytical and Quantitative Cytology and Histology 10 (1988): 375-382.

36. Michiels JJ., et al. "The 2001 world health organization and updated european clinical and pathological criteria for the diagnosis classification and staging of the Philadelphia-negative chronic myeloproliferative disorders". Seminars in Thrombosis and Hemostasis 32 (2006): 307-340.

37. Dameshek W. "Some speculations on the myeloproliferative syndromes". Blood 6 (1951): 372-375.

38. Michiels JJ., et al. "PVSG and WHO vs European Clinical, Molecular and Pathological (ECMP) criteria for prefibrotic myeloproliferative neoplasms". World Journal of Hematology 2 (2013): 71-88.

39. Michiels JJ., et al. "Extension of 2016 World Health Organization (WHO) classification and a new set of clinical, laboratory, molecular and pathological criteria for the diagnosis of myeloproliferative neoplasms: from Dameshek to Vainchenker, Green and Kralovics". Eur Med J 2.2 (2017a):72-81.

40. De Raeve H., et al. "Novel Clinical, Laboratory, Molecular and Pathological (2018 CLMP) Criteriafor the Differential Diagnosis of three Distinct JAK2, CALR and MPL Mutated Myeloproliferative Neoplasms: The Role of Driver Mutation Analysis and Bone Marrow Histology". International Journal of Cancer Research and Therapy 3.2 (2018):1-12.

\section{Volume 3 Issue 11 November 2019}

(c) All rights are reserved by Jan Jacques Michiels., et al. 